\title{
Análise dos Resultados do Atendimento ao Paciente com Infarto Agudo do Miocárdio com Supradesnivelamento do Segmento ST nos Períodos Diurno e Noturno
}

\author{
Gustavo Oliveira de Albuquerque ${ }^{1}$, Eduardo Szuster ${ }^{1}$, Luiz Carlos Teles Corrêa ${ }^{1}$, Eugênio Goulart ${ }^{1}$, \\ Antônio César Souza', Antônio Luiz de O. A. Sobrinho', Maurício de Rezende Barbosa'
}

\section{RESUMO}

Introdução: A angioplastia coronariana primária com implante de stents é considerada o melhor método de tratamento no infarto agudo do miocárdio com supradesnivelamento do segmento ST (IAMCSST), com resultados mais favoráveis e menor incidência de complicações quando comparada a outras formas de tratamento. Avaliamos a eficácia do atendimento nos período diurno e noturno e a evolução hospitalar desses pacientes. Método: Analisamos, de forma retrospectiva, os dados de 274 pacientes submetidos a angioplastia primária no período compreendido entre julho de 2004 e agosto de 2008, divididos em dois grupos: os atendidos no período diurno e os atendidos no período noturno. Foram analisados as características clínicas e angiográficas, o tempo porta-balão, o sucesso angiográfico primário e a ocorrência de complicações. Resultados: Dos 274 pacientes analisados, 186 (67,8\%) foram atendidos no período diurno e $88(32,2 \%)$, no período noturno. As características clínicas e angiográficas foram semelhantes em ambos os períodos. O tempo portabalão alcançado no período noturno $(64,1$ minutos) foi menor que o do período diurno (72,5 minutos) $(P=0,02)$. O sucesso angiográfico foi semelhante (93\%) em ambos os turnos. Houve maior número de complicações maiores no período noturno, à custa de acidente vascular cerebral ( $0 \%$ vs. $3,4 \% ; P=0,032)$, reinfarto $(1 \%$ vs. $5,6 \% ; P=0,037)$ e revascularização da lesão-alvo ( $1 \%$ vs. $5,6 \%$; $P=0,037)$. Conclusão: $O$ atendimento realizado fora do período diurno em nosso serviço foi rápido e eficaz, com índices de sucesso angiográfico similares aos do atendimento em horas regulares. As complicações clínicas, no entanto, foram maiores nos pacientes tratados no período noturno,

\section{ABSTRACT}

Treatment Outcome of Patients with Acute Myocardial Infarction and ST Segment Elevation in Routine Duty Hours and Off-Hours

Background: Primary coronary angioplasty with stent implantation has been considered the treatment of choice in acute myocardial infarction with ST segment elevation, leading to more favorable outcomes and lower incidence of complications when compared to other treatments. The efficacy of patient care in routine duty hours and off-hours and the in-hospital course of these patients were analyzed. Methods: Two hundred and seventy-four patients undergoing primary angioplasty between July 2004 and August 2008 were retrospectively analyzed and divided into two groups: those treated during routine duty hours and those treated during off-hours. Clinical and angiographic characteristics, door-to-balloon time, primary angiographic success, and the occurrence of complications were analyzed. Results: Of 274 patients studied, $186(67.8 \%)$ were treated during routine duty hours and $88(32.2 \%)$ were treated during off-hours. Clinical and angiographic characteristics were similar in both periods. Door-to-balloon time at night (64.1 minutes) was lower than at daytime (72.5 minutes). Primary angiographic success was similar (93\%) in both shifts. There was a greater number of major complications at night due to stroke $(0 \%$ vs. $3.4 \%$; $\mathrm{P}=0.032)$, reinfarction $(1 \%$ vs. $5.6 \% ; \mathrm{P}=0.037)$ and target lesion revascularization $(1 \%$ vs. $5.6 \% ; P=0.037)$. Conclusion: At our service, medical care provided in the off-hours was quick and effective with angiographic success rates similar to those obtained in routine duty hours. However, clinical com-

\footnotetext{
1 Hospital Biocor - Belo Horizonte, MG, Brasil.

Correspondência: Gustavo Oliveira de Albuquerque. Rua Eli Seabra

Filho, 157/301 - Buritis - Belo Horizonte, MG, Brasil - CEP 30575-740

E-mail: goalbuquerque@gmail.com

Recebido em: 5/1/2009 - Aceito em: 22/3/2009
} 
Albuquerque GO, et al. Análise dos Resultados do Atendimento ao Paciente com Infarto Agudo do Miocárdio com Supradesnivelamento do Segmento ST nos Períodos Diurno e Noturno. Rev Bras Cardiol Invas. 2009;17(1):52-7.

possivelmente influenciadas por fatores não avaliados neste estudo.

DESCRITORES: Infarto do miocárdio. Angioplastia. Contenedores. Fatores de tempo.

É reconhecido que o infarto agudo do miocárdio com supradesnivelamento do segmento ST (IAMCSST) tem forte impacto socioeconômico, pela limitação que essa doença impõe a seus portadores. Nos Estados Unidos, segundo dados de 2006, $700 \mathrm{mil}$ pacientes sofrem novos ataques cardíacos anualmente' No Brasil, ocorrem cerca de 350 mil novos casos por ano, segundo as últimas atualizações do Departamento de Informática do Sistema Único de Saúde (DATASUS) $2004^{2}$.

Ao lado da angina instável, do infarto agudo do miocárdio sem supradesnivelamento do segmento ST e da morte súbita, o IAMCSST completa o heterogêneo espectro das síndromes coronarianas agudas. A oclusão total de uma artéria coronária epicárdica, determinada pela presença de trombo oclusivo subjacente a uma placa aterosclerótica, na maioria das vezes, é o substrato fisiopatológico do IAMCSST ${ }^{3}$. Essa síndrome decorre de uma complexa cadeia de acontecimentos, tendo como base a chamada placa aterosclerótica vulnerável, composta por um núcleo denso de lípides, capa fibrótica delgada, infiltrada por células inflamatórias e com modificações degenerativas da matriz extracelular. O tratamento tem como base a reperfusão química (fibrinolíticos) ou mecânica (angioplastia). Segundo as diretrizes americanas de 2005, a restauração do fluxo normal da artéria obstruída, por meio da intervenção coronariana percutânea ou de fibrinolíticos, é determinante para uma boa evolução, tanto precoce como tardia, dos pacientes com IAMCSST ${ }^{4-6}$.

No início da década de 1980, houve grandes avanços terapêuticos com o uso rotineiro tanto dos fibrinolíticos como da angioplastia coronariana, inicialmente com balão e posteriormente com stents ${ }^{7}$. Atualmente a indicação preferencial é para tratamento intervencionista, desde que infraestrutura apropriada esteja disponível, em decorrência da superioridade da angioplastia primária constatada em relação aos fibrino$\operatorname{líticos}^{2,8}$. Resultados da comparação entre intervenção coronariana percutânea com implante de stent e fibrinolíticos no tratamento do infarto agudo do miocárdio demonstram que pacientes tratados com stent evoluem com menores taxas de mortalidade, reinfarto e acidente vascular cerebral hemorrágico ${ }^{9}$.

Poucos centros dispõem de serviço de cardiologia intervencionista, e nem todos disponibilizam atendimento de urgência que funcione 24 horas por dia durante todo o ano. Sabe-se que a logística do atendi- plications were greater in patients treated in the off-hours, possibly as a result of factors not assessed in this study.

DESCRIPTORS: Myocardial infarction. Angioplasty. Stents. Time factors.

mento no laboratório de hemodinâmica no período noturno é mais complexa, visto que depende da disponibilidade do atendimento de toda uma equipe multidisciplinar (pronto-socorro, médico hemodinamicista, enfermagem, farmácia, internação, etc.).

Realizamos um estudo com o objetivo de avaliar os resultados dos pacientes com IAMCSST submetidos a angioplastia primária, em nosso hospital, que dispõe de serviço de intervenção coronariana percutânea primária 24 horas por dia, com a finalidade de comparar a eficácia do atendimento nos períodos diurno e noturno.

\section{MÉTODO}

O presente estudo é um registro de casos conduzido de forma transversal, realizado em um único centro médico onde a intervenção coronariana percutânea de urgência é realizada de rotina em todos os pacientes com IAMCSST. Os pacientes atendidos durante o dia foram comparados aos atendidos à noite. O período diurno foi considerado o intervalo compreendido entre as 7 horas e as 19 horas, quando toda a equipe está presente no serviço. Os finais de semana e feriados foram considerados como "período noturno", independentemente do horário de admissão do paciente no hospital.

As intervenções foram realizadas por diferentes operadores, seguindo a escala de plantão de 4 cardiologistas da equipe de hemodinâmica, sendo 3 membros titulares da Sociedade Brasileira de Hemodinâmica e Cardiologia Intervencionista $(\mathrm{SBHCl})$ e 1 médico sócio aspirante. O médico mais antigo tem 30 anos de certificação pela $\mathrm{SBHCl}$ e o mais novo, 9 anos. Os 4 plantonistas foram os mesmos que realizavam os procedimentos de urgência e eletivos durante o período diurno.

No serviço em análise, há uma média de, aproximadamente, 60 angioplastias primárias por ano na vigência de IAMCSST. Foram incluídos 274 pacientes consecutivos submetidos a intervenção coronariana percutânea primária no serviço no período de julho de 2004 a agosto de 2008. A maior parte dos pacientes foi atendida no pronto-socorro e encaminhada para o serviço de hemodinâmica para coronariografia de urgência e angioplastia primária. Uma parcela menor de pacientes foi encaminhada diretamente ao setor de hemodinâmica (encaminhados de outros centros já 
com diagnóstico prévio) e outros poucos pacientes já se encontravam internados em outras dependências do hospital. O limite de retardo aceito para intervenção coronariana percutânea primária foi de no máximo 24 horas (acima de 12 horas foram tratados os que apresentavam evidência de viabilidade miocárdica, angina refratária ou choque cardiogênico). Todos os pacientes fizeram uso de terapia antiplaquetária dupla pré-intervenção, com dose de ataque de $325 \mathrm{mg}$ a $500 \mathrm{mg}$ de aspirina e de $300 \mathrm{mg}$ a $600 \mathrm{mg}$ de clopidogrel no momento do diagnóstico de infarto agudo do miocárdio. Como rotina, utilizamos a heparina não-fracionada na dose de 70 a $100 \mathrm{UI} / \mathrm{kg}$ de peso em forma de bolus no momento da intervenção coronariana percutânea. Nitrato intracoronariano ou sistêmico e inibidores da glicoproteína IIb/IIla (tirofiban) foram utilizados em casos selecionados ${ }^{10,11}$, de acordo com avaliação do hemodinamicista. A maioria dos casos recebeu stents não-farmacológicos e em poucos casos foi realizada intervenção coronariana percutânea primária somente com o balão. Após a angioplastia, os pacientes foram encaminhados para a unidade coronariana e receberam tratamento conforme protocolo estabelecido para IAMCSST.

Comparamos as características clínicas e angiográficas, o tempo porta-balão, o sucesso angiográfico, e a ocorrência de complicações clínicas nos dois períodos. Os dados foram coletados de um banco de informações preexistente do setor de hemodinâmica, preservando a identidade dos pacientes. O conteúdo capturado foi tabulado em uma planilha do Excel e, posteriormente, analisado em um programa estatístico Epi-Info. As variáveis categóricas foram expressas em porcentuais e comparadas com o teste de qui-quadra- do ou o teste exato de Fisher, quando apropriado. As variáveis contínuas foram expressas em médias e analisadas pelo teste $t$ de Student. Foi considerado significativo $\mathrm{P}<0,05$.

\section{RESULTADOS}

Foram analisados os resultados de 274 pacientes consecutivos com IAMCSST, submetidos a angioplastia primária no período de julho de 2004 a agosto de 2008, $186(67,9 \%)$ tratados no período diurno e 88 $(32,1 \%)$ tratados no período noturno.

Em relação às características clínicas, não observamos diferenças entre os grupos. Predominaram pacientes do sexo masculino (78\%), com média de idade de 62,9 anos (30 a 95 anos), 14,9\% portadores de diabetes melito e 79,6\% admitidos em Killip I (Tabela 1).

Quanto às características angiográficas, também não notamos diferenças entre os grupos (Tabela 2). Mais da metade dos pacientes era portadora de lesões em dois ou três vasos e ventriculografia esquerda foi realizada em $78,2 \%$ dos pacientes. Pouco mais de $20 \%$ dos que dispunham da avaliação contrastada do ventrículo esquerdo tinham disfunção ventricular grave. O vaso mais frequentemente tratado foi a artéria descendente anterior $(38,7 \%$ vs. $37,5 \%)$ e somente uma minoria tratou enxertos de safena $(3,2 \%$ vs. $2,2 \%$ ).

O tempo porta-balão foi de 68,3 minutos, mais curto no período noturno (64,1 minutos vs. 72,5 minutos; $P=0,02$ ). Mais de dois terços dos pacientes demonstrou oclusão total ou funcional do vaso (fluxos TIMI 0 e 1) na angiografia inicial, achado semelhante

TABELA 1

Características clínicas dos 274 pacientes que realizaram angioplastia primária na vigência de infarto agudo do miocárdio com supradesnivelamento do segmento ST

\begin{tabular}{|c|c|c|c|c|}
\hline & Total $(n=274)$ & Diurno $(n=186)$ & Noturno $(\mathrm{n}=88)$ & $\mathbf{P}$ \\
\hline Sexo masculino & $212(78 \%)$ & $142(76,3 \%)$ & $70(79,5 \%)$ & 0,43 \\
\hline Idade, anos & 62,9 & 64,7 & 61,1 & 0,12 \\
\hline$>75$ anos & $64(23,3 \%)$ & $45(24,19 \%)$ & $19(21,59 \%)$ & 0,20 \\
\hline Diabetes melito & 41 (14,9\%) & $30(16,1 \%)$ & 11 (12,5\%) & 0,54 \\
\hline HAS & $177(64,5 \%)$ & $126(67,7 \%)$ & $51(57,9 \%)$ & 0,15 \\
\hline Dislipidemia & $151(55,1 \%)$ & $106(56,9 \%)$ & $45(51,1 \%)$ & 0,43 \\
\hline Tabagismo & $65(23,7 \%)$ & 39 (20,9\%) & $26(29,5 \%)$ & 0,16 \\
\hline ICP prévia & $31(11,3 \%)$ & $24(12,9 \%)$ & $7(7,9 \%)$ & 0,31 \\
\hline RM prévia & $15(5,4 \%)$ & $10(5,3 \%)$ & $5(5,6 \%)$ & $>0,99$ \\
\hline Killip & & & & 0,83 \\
\hline I & $218(79,6 \%)$ & $149(80,1 \%)$ & $69(78,4 \%)$ & \\
\hline ॥ & $35(12,8 \%)$ & $24(12,9 \%)$ & $11(12,5 \%)$ & \\
\hline III-IV & $21(7,6 \%)$ & $13(7 \%)$ & $8(9,1 \%)$ & \\
\hline
\end{tabular}

HAS = hipertensão arterial sistêmica; ICP = intervenção coronariana percutânea; $\mathrm{n}=$ número de pacientes; $\mathrm{RM}$ = revascularização miocárdica. 
TABELA 2

Características angiográficas e do procedimento dos 274 pacientes que realizaram angioplastia primária na vigência de infarto agudo do miocárdio com supradesnivelamento do segmento ST

\begin{tabular}{|c|c|c|c|}
\hline & Diurno $(n=186)$ & Noturno $(n=88)$ & $\mathbf{P}$ \\
\hline Número de vasos acometidos & & & 0,81 \\
\hline 1 & $78(41,9 \%)$ & $35(39,8 \%)$ & \\
\hline 2 & $50(26,8 \%)$ & $27(30,7 \%)$ & \\
\hline 3 & $58(31,7 \%)$ & $26(29,5 \%)$ & \\
\hline Comprometimento da FV & & & 0,51 \\
\hline Normal/Leve & $65(34,9 \%)$ & $31(35,3 \%)$ & \\
\hline Moderado & $42(22,5 \%)$ & $18(21,2 \%$ & \\
\hline Grave & $37(20 \%)$ & $20(23,1 \%)$ & \\
\hline Vaso tratado & & & 0,48 \\
\hline DA & $72(38,7 \%)$ & $33(37,5 \%)$ & \\
\hline$C X$ & $50(26,8 \%)$ & $24(27,2 \%)$ & \\
\hline $\mathrm{CD}$ & $55(29,5 \%)$ & $28(31,8 \%)$ & \\
\hline TCE & 0 & 0 & \\
\hline Ponte de safena & $6(3,2 \%)$ & $2(2,2 \%)$ & \\
\hline Mamária & $3(1,6 \%)$ & $1(1,1 \%)$ & \\
\hline Tempo porta-balão & $72,5 \mathrm{~min}$ & $64,1 \mathrm{~min}$ & 0,02 \\
\hline Fluxo TIMI pré & & & 0,63 \\
\hline 0 & $77(41,3 \%)$ & $40(45,4 \%)$ & \\
\hline 1 & $59(31,7 \%)$ & $29(32,9 \%)$ & \\
\hline 2 & $50(26,8 \%)$ & $19(21,5 \%)$ & \\
\hline 3 & 0 & 0 & \\
\hline Sucesso angiográfico & $173(93 \%)$ & $82(93 \%)$ & NA \\
\hline ATC com balão & $15(8,0 \%)$ & $8(9,0 \%)$ & 0,81 \\
\hline Fluxo TIMI pós & & & 0,98 \\
\hline 0 & $5(2,6 \%)$ & $3(3,4 \%)$ & \\
\hline 1 & $5(2,6 \%)$ & $2(2,2 \%)$ & \\
\hline 2 & $2(1 \%)$ & $1(1,1 \%)$ & \\
\hline 3 & $174(93,5 \%)$ & $82(93,1 \%)$ & \\
\hline
\end{tabular}

ATC = angioplastia transluminal coronariana; $C D=$ artéria coronária direita; $C X=$ artéria circunflexa; $D A=$ artéria descendente anterior; $\mathrm{FV}=$ função ventricular; $\mathrm{n}=$ número de pacientes; TCE = tronco da artéria coronária esquerda.

entre os grupos. O sucesso angiográfico foi idêntico (93\%) nos dois períodos e o fluxo TIMI 3 final foi obtido em $93,5 \%$ e 93,1\% dos casos, respectivamente.

A Tabela 3 mostra a incidência de complicações maiores hospitalares. Ocorreram 21 óbitos $(7,7 \%$ do total), 11/186 (5,9\%) no período diurno e 10/88 (11,3\%) no período noturno $(\mathrm{P}=0,18)$. Os acidentes vasculares cerebrais acometeram 3 pacientes, todos do grupo de atendimento noturno $(\mathrm{P}=0,032)$. A trombose do stent ocorreu em 7 pacientes, sendo $2(1 \%)$ no período diurno e $5(5,6 \%)$ no noturno $(P=0,037)$, todos os casos com manifestação clínica de reinfarto e tratados percutaneamente. Complicações vasculares (sangramento maior, pseudoaneurisma) foram observadas em 3 pacientes, sem diferença estatística nos diferentes turnos ( $1 \%$ vs. $1,1 \%)$.

\section{DISCUSSÃO}

O presente estudo demonstrou que: 1. o perfil tanto clínico como angiográfico dos pacientes submetidos a angioplastia primária não diferiu nos períodos diurno e noturno; 2 o atendimento realizado no período noturno foi mais rápido que o diurno (tempo porta-balão 6,4 minutos menor); 3. apesar do sucesso angiográfico similar, ocorreu maior número de complicações no período noturno.

Nosso centro é considerado referência em cardiologia para outros hospitais menores e cidades vizinhas e a não-seleção dos pacientes encaminhados pode explicar a ausência de diferenças nas características clínicas e angiográficas nos admitidos durante o dia ou à noite. 
TABELA 3

Evolução clínica hospitalar

\begin{tabular}{lccc}
\hline & Diurno $(\mathbf{n}=\mathbf{1 8 6})$ & Noturno $(\mathbf{n}=\mathbf{8 8})$ & $\mathbf{P}$ \\
\hline Óbito & $11(5,9 \%)$ & $10(11,3 \%)$ & 0,18 \\
Acidente vascular cerebral & 0 & $3(3,4 \%)$ & 0,032 \\
Reinfarto & $2(1 \%)$ & $5(5,6 \%)$ & 0,037 \\
Revascularização do vaso-alvo & $2(1 \%)$ & $5(5,6 \%)$ & 0,037 \\
Trombose do stent & $2(1 \%)$ & $5(5,6 \%)$ & 0,037 \\
Complicações vasculares & $2(1 \%)$ & $1(1,1 \%)$ & $>0,99$ \\
\hline
\end{tabular}

$\mathrm{n}=$ número de pacientes

O tempo porta-balão tem fundamental importância na avaliação do atendimento prestado em serviços de hemodinâmica. É considerado o período entre a entrada do paciente no pronto-socorro e a reabertura do vaso ocluído e deve ser o mais curto possível, pois quanto menor o tempo de sofrimento miocárdico, melhor o prognóstico do paciente $\mathrm{e}^{12-14}$. O tempo portabalão ideal é menor ou igual a 90 minutos $^{4}$. Ele depende de uma série de fatores, desde a eficiência do diagnóstico no pronto-socorro até a rapidez com que o hemodinamicista atua desobstruindo o vaso. Nosso tempo porta-balão foi curto nos dois períodos analisados, mas 8,4 minutos menor no período noturno. Essa diferença poderia ser explicada pela menor movimentação do pronto-socorro no período noturno; pela chegada rápida dos hemodinamicistas envolvidos, que residem perto do hospital; e pela completa disponibilidade das salas de hemodinâmica para as emergências noturnas com toda a infraestrutura pronta, inclusive enfermeiros e pessoal técnico especializado.

O IAMCSST, a apresentação mais letal da síndrome coronariana aguda, apresenta altos índices de óbitos e complicações graves quando não tratado adequadamente ou quando tratado tardiamente. No presente estudo, observamos que o sucesso angiográfico primário nos dois períodos analisados foi satisfatório (93\%). A análise das complicações demonstrou diferença na incidência de acidente vascular cerebral e na trombose do stent, e suas consequências (reinfarto e revascularização de urgência da lesão-alvo), com piores resultados para o período noturno. Além disso, ocorreu o dobro de óbitos no período noturno (5,9\% vs. 11,3\%), embora sem significância estatística $(P=0,18)$. Esses dados são concordantes com a literatura, que mostra que os pacientes atendidos durante o período offhour (noite, feriado e final de semana) têm piores resultados, por diferenças na logística de apresentação/admissão ao hospital, no perfil social e clínico, e no desempenho das equipes de atendimento ${ }^{15}$. É possível que variáveis não avaliadas, como tempo de isquemia miocárdica total mais prolongado, decorrente da demora dos pacientes em chegar ao hospital no período noturno, entre outras, possam ter influenciado a pior evolução nesse período.

\section{CONCLUSÕES}

$\mathrm{O}$ atendimento realizado fora do período diurno em nosso serviço foi rápido e eficaz, com índices de sucesso angiográfico similares aos do atendimento nas horas regulares. As complicações clínicas, no entanto, foram maiores nos pacientes tratados no período noturno, possivelmente influenciadas por fatores não avaliados neste estudo.

\section{CONFLITO DE INTERESSES}

Os autores declararam inexistência de conflito de interesses.

\section{REFERÊNCIAS BIBLIOGRÁFICAS}

1. American Heart Association. Heart disease and stroke statistic - 2006 update: a report from the American Heart Association Statistics Committee and Stroke Statistics Subcommittee. Circulation. 2006;113(6):e85-151.

2. Ribeiro EE, Martinez EE. Hemodinâmica e cardiologia intervencionista: abordagem clínica. São Paulo: Manole; 2008. p.191-211.

3. Keeley EC, Grines CL. Primary coronary intervention for acute myocardial infarction. JAMA. 2004;291(6):736-9.

4. ACC/AHA/SCAI 2005 Guidelines Update for Percutaneous Coronary Intervention: a report of the American College of Cardiology/American Heart Association Task Force on Practice Guidelines (ACC/AHA/SCAI Writing Committee to Update the 2001 Guidelines for Percutaneous Coronary Intervention). Disponível em: http://www.americanheart.org/. Acesso em novembro de 2008.

5. Van de Werf F, Bax J, Betriu A, Blomstrom-Lundqvist C, Crea $\mathrm{F}$, Falk $\mathrm{V}$, et al. Management of acute myocardial infarction in patients presenting with persistent ST-segment elevation: the Task Force on the Management of ST-Segment Elevation Acute Myocardial Infarction of the European Society of Cardiology. Eur Heart J. 2008;29(23):2909-45.

6. Masoudi FA, Bonow RO, Brindis RG, Cannon CP, Debuhr J, Fitzgerald S, et al. ACC/AHA 2008 statement on Performance Measurement and Reperfusion Therapy: a report of the ACC/ AHA Task Force on Performance Measures (Work Group to address the challenges of Performance Measurement and Reperfusion Therapy). J Am Coll Cardiol. 2008;52(24):2100-12.

7. Kukreja N, Onuma Y, Garcia-Garcia H, Daeman J, van Domburg R, Serruys PW. Primary percutaneous coronary intervention for acute myocardial infarction: long-term outcome after baremetal and drug-eluting stent implantation. Circ Cardiovasc Intervent. 2008;1:103-10. 

do Segmento ST nos Períodos Diurno e Noturno. Rev Bras Cardiol Invas. 2009;17(1):52-7.

8. Allen LA, O'Donnell CJ, Camargo Jr CA, Giugliano RP, LloydJones DM. Comparison of long-term mortality across the spectrum of acute coronary syndromes. Am Heart J. 2006; 151(5):1065-71.

9. Keeley EC, Boura JA, Grines CL. Primary angioplasty versus intravenous thombolytic therapy for acute myocardial infarction: a quantitative review of 23 randomized trials. Lancet. 2003;361(9351):13-20.

10. Shen J, Zhang Q, Zhang RY, Zhang JS, Hu J, Yang ZK, et al. Clinical benefits of adjunctive tirofiban therapy in patients with acute ST-segment elevation myocardial infarction undergoing primary percutaneous coronary intervention. Coron Artery Dis. 2008;19(4):271-7.

11. Van't Hof AW, Ten Berg J, Heestermans T, Dill T, Funck RC, van Werkum W, et al. Prehospital initiation of tirofiban in patients with ST-elevation myocardial infarction undergoing primary angioplasty (on-time 2): a multicenter, double-blind, randomized controlled trial. Lancet. 2008;372(9638):537-46.

12. Antman EM. Time is muscle: translation into practice. J Am Coll Cardiol. 2008;52(15):1216-21.

13. Braunwald E, Antman EM, Beasley JW, Califf RM, Cheitlin MD, Hochman JS, et al.; American College of Cardiology;
American Heart Association. Committee on the Management of Patients with Unstable Angina. ACC/AHA 2002 guideline update for the management of patients with unstable angina and non-ST-segment elevation myocardial infarction - summary article: a report of the American College of Cardiology/ American Heart Association task force on practice guidelines (Committee on the Management of Patients with Unstable Angina). J Am Coll Cardiol. 2002;40(7):1366-74.

14. Antman EM, Anbe DT, Armstrong PW, Bates ER, Green LA, Hand $M$, et al.; American College of Cardiology; American Heart Association Task Force on Practice Guidelines; Canadian Cardiovascular Society. ACC/AHA guidelines for the management of patients with ST-elevation myocardial infarction: a report of the American College of Cardiology/American Heart Association Task Force on Practice Guidelines (Committee to Revise the 1999 Guidelines for the Management of Patients with Acute Myocardial Infarction). Circulation. 2004;110(9):e82-292.

15. Jneid H, Fonarow GC, Cannon CP, Palacios IF, Kilic T, Moukarbel GV, et al.; Get With the Guidelines Steering Committee and Investigators. Impact of time of presentation on the care and outcomes of acute myocardial infarction. Circulation. 2008;117(19):2502-9. 